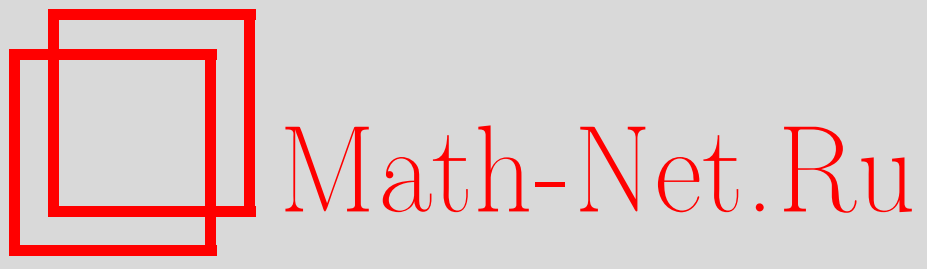

П. И. Кацыло, В. Л. Попов, О неподвижных точках алгебраических действий на $\mathbb{C}^{n}$, Функи. анализ и его прил., 2000, том 34, выпуск 1, 41-50

DOI: https://doi.org/10.4213/faa281

Использование Общероссийского математического портала MathNet.Ru подразумевает, что вы прочитали и согласны с пользовательским соглашением

http: //www . mathnet.ru/rus/agreement

Параметры загрузки:

IP : 54.198 .55 .26

26 апреля 2023 г., 06:33:25

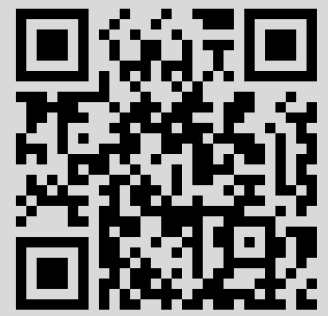


Функциональньй анализ и его приложения

2000, т. 34, вып. 1, с. 41-50

УДК 512.743

\title{
О неподвижных точках алгебраических действий на $\mathbb{C}^{n}$ *
}

\author{
(с) 2000. П. И. КАЦЫЛО, В. Л. ПоПОВ
}

1. Несмотря на значительные успехи современных исследований конечномерных подгрупп аффинных групп Кремоны (см. обзор [5], а также статью [2] и библиографию к ней), следующая центральная проблема не решена до сих пор: у любого ли алгебраического действия комплексной редуктивной группы $G$ на аффинном пространстве $\mathbb{C}^{n}$ существует неподвижная точка?

Ожидается, что ответ отрицательный. Действие без неподвижных точек было бы, помимо прочего, и примером алгебраического голоморфно нелинеаризуемого действия (все известные до сих пор алгебраически нелинеаризуемые действия голоморфно линеаризуемы).

2. В качестве возможного кандидата, доставляющего отрицательный ответ, специалисты уже довольно давно рассматривают действие, описанное ниже. Согласно фольклору, его конструкция принадлежит Д. Луне. В ней $G=\mathrm{SL}_{2}(\mathbb{C})$ и $n=8$. По-видимому, этот выбор группы и размерности инспирирован работой [6], где установлено, что дифференцируемые действия группы $\mathrm{SO}_{3}(\mathbb{R})$ на $d$-мерном диске имеют неподвижную точку при $d \leqslant 7$, а при $d=8$ существуют действия без неподвижных точек: дело в том, что стабилизаторы и страты в предложенной Д. Луной конструкции в точности такие же, как и в построенном в [6] действии без неподвижных точек.

3. Чтобы описать эту конструкцию, обозначим через $V_{d}$ пространство бинарных форм степени $d$ от переменных $x$ и $y$, на котором линейными заменами переменных естественно действует группа $G=\mathrm{SL}_{2}(\mathbb{C})$. Через $\left\langle f_{1}, \ldots, f_{m}\right\rangle$ будем обозначать линейную оболочку форм $f_{1}, \ldots, f_{m} \in V_{d}$.

Рассмотрим в $V_{8}$ орбиту $\mathscr{O}$ точки $x^{4} y^{4}$ и следующую гиперплоскость $N$ в пространстве, нормальном к $\mathscr{O}$ в этой точке, инвариантную относительно стабилизатора этой точки:

$$
N:=x^{4} y^{4}+\left\langle x^{8}, x^{7} y, x^{6} y^{2}, x^{2} y^{6}, x y^{7}, y^{8}\right\rangle .
$$

Пусть теперь $L:=G \cdot N-$ множество в $V_{8}$, полученное «разнесением»гиперплоскости $N$ с помощью $G$. Оно является образом соответствующего подрасслоения нормального расслоения над $\mathscr{O}$ при естественном морфизме в $V_{8}$, откуда следует, что $L$ конструктивно и инвариантно, а его замыкание в $V_{8}$ является неприводимой гиперповерхностью.

Действие группы $G$ на $L$ и является упомянутым кандидатом. Так как 0 - единственная неподвижная точка в $V_{8}$ и $0 \notin N$, то $G$ действует на $L$ без неподвижных точек. Вопрос состоит в том, является ли $L$ локально замкнутым подмножеством в $V_{8}$, изоморфным аффинному пространству, или нет.

* Работа выполнена при поддержке CRDF, грант RM1-206, и INTAS, грант INTAS-OPEN97-1570. 
4. Мы покажем здесь, что ответ отрицательный и, следовательно, рассмотрение действия группы $G$ на $L$ не ведет к решению проблемы неподвижных точек. В действительности мы доказываем гораздо больше. А именно, с множеством $L$ естественно связаны два многообразия - его открытое ядро $L^{0}:=$ $\bar{L} \backslash(\overline{\bar{L} \backslash L})=L \backslash(\overline{\bar{L} \backslash L})$ (здесь и далее черта, как обычно, обозначает замыкание) и его замыкание $\bar{L}$. Ввиду отрицательного ответа на исходный вопрос естественно спросить, не будет ли тогда действием без неподвижных точек на аффинном пространстве действие группы $G$ на $L^{0}$ или $\bar{L}$.

Условимся, что любая константа является корнем нулевой формы, кратность которого равна $+\infty$. Ниже мы даем явное описание $\bar{L}$ и $L^{0}$.

Teорема 1. (i) Множество $\bar{L}$ совпадает $c L \cup D$, аде

$$
D:=\left\{g \in V_{8} \mid g \text { имеет корень кратности } \geqslant 3\right\} \text {. }
$$

(ii) Множество $D$ замкнуто, неприводимо $u \operatorname{codim}_{\bar{L}} D=1$.

(iii) Множество $\bar{L}$ содержит 0 , единственную неподвижную точку в $V_{8}$.

(vi) Множество $L \cap D$ непусто.

ЗАМЕчАНИЕ 1. Можно показать, что многообразие $\bar{L}$ имеет особенности: например, $x^{4} y^{4}$ - его особая точка.

ЗАмечАниЕ 2. Пользуясь техникой детерминантов комплексов (см. [4]), можно показать, что гиперповерхность $\bar{L}$ задается в $V_{8}$ уравнением степени 27.

Tеорема 2. Множество $L^{0}$ совпадает $c L \backslash D=\bar{L} \backslash D$.

Поскольку локально замкнутое подмножество совпадает со своим открытым ядром, из теорем 1 и 2 немедленно вытекает отрицательный ответ на исходный вопрос:

СлЕдСТВИЕ. Множество L не является открытым подмножеством в своем замыкании.

Согласно п. (iii) теоремы $1, \bar{L}$ тоже не является примером действия на аффинном пространстве без неподвижных точек. Мы показываем, что таким примером не является и $L^{0}$. Более того, мы доказываем следующее общее утверждение, из которого вытекает, что, вообще, никакое $G$-инвариантное открытое подмножество в $L^{0}$ не является таким примером:

ТЕОремА 3. Многообразие $L^{0}$ не содержит $G$-инвариантных открытых нормальных подмногообразий с тривиальной группой Пикара, на которьх нет непостоянных обратимых регулярньх функиий.

СлЕдСТВИЕ. Многообразие $L^{0}$ не содержит G-инвариантных открытых подмногообразий, изоморфных аффинному пространству. В частности, само $L^{0}$ не изоморфно аффинному пространству.

5. Доказательства основаны на вспомогательной конструкции. Чтобы описать ее, введем дополнительные обозначения:

$\mathbb{P}\left(V_{d}\right)$ - проективизация векторного пространства $V_{d}$, снабженная естественным действием группы $G$;

$[u]$ — точка в $\mathbb{P}\left(V_{d}\right)$, соответствующая прямой $\langle u\rangle$, где $u \in V_{d}, u \neq 0$; 
$(f, g)_{i}$ - значение $i$-го трансвектанта на бинарных формах $f \in V_{d}$ и $g \in V_{e}$. Нам понадобится его явный вид (см. [1]):

$$
(f, g)_{i}= \begin{cases}\frac{(d-i) !(e-i) !}{d ! e !} \sum_{j=0}^{i}(-1)^{j}\left(\begin{array}{l}
i \\
j
\end{array}\right) \frac{\partial^{i} f}{\partial x^{i-j} \partial y^{j}} \frac{\partial^{i} g}{\partial x^{j} \partial y^{i-j}}, & i \leqslant \min \{d, e\}, \\
0, & i>\min \{d, e\} .\end{cases}
$$

Пусть $i \leqslant \min \{d, e\} ;$ трансвектант $V_{d} \times V_{e} \rightarrow V_{d+e-2 i},(f, g) \mapsto(f, g)_{i}$, является билинейным $G$-эквивариантным морфизмом.

6. Рассмотрим теперь в прямом произведении $\mathbb{P}\left(V_{2}\right) \times V_{8}$ замкнутую подсхему

$$
Z:=\left\{([f], g) \in \mathbb{P}\left(V_{2}\right) \times V_{8} \mid 35\left(f^{3}, g\right)_{6}=2(f, f)_{2} f\right\}
$$

и обозначим через

$$
\mathbb{P}\left(V_{2}\right) \stackrel{\pi_{2}}{\longleftarrow} Z \stackrel{\pi_{8}}{\longrightarrow} V_{8}
$$

естественные проекции.

Ввиду полноты многообразия $\mathbb{P}\left(V_{2}\right)$ морфизм $\pi_{8}$ замкнут. Поскольку трансвектанты эквивариантны, из вида уравнения в правой части формулы (6.1) следует, что $Z$ является $G$-инвариантной подсхемой. Морфизмы $\pi_{2}, \pi_{8} G$-эквивариантны.

7. Чтобы получить информацию о геометрических свойствах схемы $Z$ и морфизмов $\pi_{2}, \pi_{8}$, нам потребуется расписать в координатах уравнение из правой части формулы (6.1).

Подставляя $f=\sum_{r=0}^{2} f_{r} x^{2-r} y^{r}$ и $g=\sum_{t=0}^{8} g_{t} x^{8-t} y^{t}$ в (6.1) и рассматривая $g_{0}, \ldots, g_{8}$ как координаты на $V_{8}$, а $f_{0}, f_{1}, f_{2}$ - как однородные координаты на $\mathbb{P}\left(V_{2}\right)$, мы получаем после вычислений, что $Z$ задается в этих координатах следуюшей системой уравнений:

$$
\left.\begin{array}{c}
280 f_{2}^{3} g_{0}-105 f_{1} f_{2}^{2} g_{1}+30\left(f_{0} f_{2}^{2}+f_{1}^{2} f_{2}\right) g_{2}-5\left(6 f_{0} f_{1} f_{2}+f_{1}^{3}\right) g_{3} \\
\quad+12\left(f_{0} f_{1}^{2}+f_{0}^{2} f_{2}\right) g_{4}-15 f_{0}^{2} f_{1} g_{5}+10 f_{0}^{3} g_{6}=8 f_{0}\left(4 f_{0} f_{2}-f_{1}^{2}\right), \\
35 f_{2}^{3} g_{1}-30 f_{1} f_{2}^{2} g_{2}+15\left(f_{0} f_{2}^{2}+f_{1}^{2} f_{2}\right) g_{3}-4\left(6 f_{0} f_{1} f_{2}+f_{1}^{3}\right) g_{4} \\
\quad+15\left(f_{0} f_{1}^{2}+f_{0}^{2} f_{2}\right) g_{5}-30 f_{0}^{2} f_{1} g_{6}+35 f_{0}^{3} g_{7}=4 f_{1}\left(4 f_{0} f_{2}-f_{1}^{2}\right), \\
10 f_{2}^{3} g_{2}-15 f_{1} f_{2}^{2} g_{3}+12\left(f_{1}^{2} f_{2}+f_{0} f_{2}^{2}\right) g_{4}-5\left(6 f_{0} f_{1} f_{2}+f_{1}^{3}\right) g_{5} \\
\quad+30\left(f_{0}^{2} f_{2}+f_{0} f_{1}^{2}\right) g_{6}-105 f_{0}^{2} f_{1} g_{7}+280 f_{0}^{3} g_{8}=8 f_{2}\left(4 f_{0} f_{2}-f_{1}^{2}\right) .
\end{array}\right\}
$$

8. Мы установим теперь несколько замечательных геометрических свойств подсхемы $Z$ и морфизмов $\pi_{2}, \pi_{8}$, которые будут использованы в доказательствах теорем. В действительности мотивировкой при подборе уравнения (6.1) были именно эти геометрические свойства.

В $\mathbb{P}\left(V_{2}\right)$ имеется ровно две $G$-орбиты: $G \cdot[x y]$ - открытая двумерная орбита точки $[x y]$ и $G \cdot\left[x^{2}\right]$ - замкнутая одномерная орбита точки $\left[x^{2}\right]$, задаваемая уравнением $4 f_{0} f_{2}-f_{1}^{2}=0$. Значит, $\pi_{2}^{-1}\left(G \cdot\left[x^{2}\right]\right)$ - замкнутое, а $\pi_{2}^{-1}(G \cdot[x y])-$ открытое $G$-инвариантные подмножества в $Z$ и $Z$ является их дизъюнктным объединением,

$$
Z=\pi_{2}^{-1}\left(G \cdot\left[x^{2}\right]\right) \sqcup \pi_{2}^{-1}(G \cdot[x y])
$$


Следующее предложение касается свойств морфизма $\pi_{2}$ :

ПРЕДЛОЖенИЕ 1. (i) Группа $G$ транзитивно действует на множестве слоев морфизма $\pi_{2}$ над точками каждой из орбит $G \cdot\left[x^{2}\right] u G \cdot[x y]$.

(ii) Все слои морфизма $\pi_{2}$ изоморфньи $\mathbb{C}^{6}$.

(iii) Множества $\pi_{2}^{-1}\left(G \cdot\left[x^{2}\right]\right)$ и $\pi_{2}^{-1}(G \cdot[x y])$ неприводимь, и их размерности равньи соответственно 7 и 8 .

(iv) Множество $Z$ неприводимо, и его размерность равна 8 .

ДоказАТельство. (i) Это следует из эквивариантности морфизма $\pi_{2}$ и транзитивности действия группы $G$ на орбитах.

(ii) Поскольку $\mathbb{P}\left(V_{2}\right)=G \cdot\left[x^{2}\right] \cup G \cdot[x y]$, из п. (i) следует, что каждый слой морфизма $\pi_{2}$ переводится подходящим преобразованием из $G$ либо в слой над $\left[x^{2}\right]$, либо в слой над $[x y]$. Поэтому описание всех слоев сводится к описанию слоев над этими двумя точками. Чтобы выяснить их структуру, воспользуемся системой уравнений (7.1).

А именно, точка $\left[x^{2}\right]$ имеет координаты $(1: 0: 0)$. После подстановки их в систему (7.1) вместо $f_{0}, f_{1}, f_{2}$ ее уравнения превращаются в $10 g_{6}=0,35 g_{7}=0$, $280 g_{8}=0$. Это означает, что

$$
\pi_{8}\left(\pi_{2}^{-1}\left(\left[x^{2}\right]\right)\right) \text { задается в } V_{8} \text { уравнениями } g_{6}=g_{7}=g_{8}=0 \text {. }
$$

Ввиду (8.2) слой $\pi_{2}^{-1}(f)$ для любой точки $f \in G \cdot\left[x^{2}\right]$ изоморфен $\mathbb{C}^{6}$.

Аналогично, поскольку точка $[x y]$ имеет координаты $(0: 1: 0)$, из (7.1) следует, что

$$
\pi_{8}\left(\pi_{2}^{-1}([x y])\right) \text { задается в } V_{8} \text { уравнениями } g_{3}=g_{5}=0, g_{4}=1 \text {. }
$$

Ввиду (8.3) слой $\pi_{2}^{-1}(f)$ для любой точки $f \in G \cdot[x y]$ тоже изоморфен $\mathbb{C}^{6}$.

(iii) Поскольку группа $G$ связна, каждая неприводимая компонента подмножества $\pi_{2}^{-1}\left(G \cdot\left[x^{2}\right]\right)$ инвариантна относительно $G$. Любой слой морфизма $\pi_{2}$ : $\pi_{2}^{-1}\left(G \cdot\left[x^{2}\right]\right) \rightarrow G \cdot\left[x^{2}\right]$, будучи, согласно п. (ii), неприводимым, целиком лежит в некоторой такой неприводимой компоненте. Ввиду п. (i) отсюда следует, что множество $\pi_{2}^{-1}\left(G \cdot\left[x^{2}\right]\right)$ неприводимо. Для $\pi_{2}^{-1}(G \cdot[x y])$ доказательство аналогично.

Утверждение о размерностях вытекает из п. (ii), равенств $\operatorname{dim}\left(G \cdot\left[x^{2}\right]\right)=1$, $\operatorname{dim}(G \cdot[x y])=2$ и теоремы о слоях морфизма.

(iv) Так как подсхема $Z$ в пространстве $\mathbb{P}\left(V_{2}\right) \times V_{8}$ задается тремя уравнениями (см. (7.1)), то размерность каждой ее неприводимой компоненты не меньше $\operatorname{dim}\left(\mathbb{P}\left(V_{2}\right) \times V_{8}\right)-3=8$. Утверждение вытекает теперь из п. (iii) и формулы (8.1).

9. Следующее утверждение касается свойств морфизма $\pi_{8}$ :

ПРЕДЛОЖЕНИЕ 2. (i) $\pi_{8}\left(\pi_{2}^{-1}(G \cdot[x y])\right)=L$.

(ii) Множества $\pi_{8}(Z)$ и $\pi_{8}\left(\pi_{2}^{-1}\left(G \cdot\left[x^{2}\right]\right)\right)$ замкнуты в $V_{8}$ и неприводимьл.

(iii) $\pi_{8}\left(\pi_{2}^{-1}\left(G \cdot\left[x^{2}\right]\right)\right)=D(c \mathcal{M} .(4.1))$.

(iv) $\pi_{8}(Z)=\bar{L}=L \cup D$.

(v) Слой $\pi_{8}^{-1}(g)$ является одной точкой для форм $g$ из некоторого непустого открытого подмножества в $\bar{L}$.

(vi) $\operatorname{dim} \bar{L}=8, \operatorname{dim} D=7$. 
ДокаЗАТЕльство. (i) Это следует из (8.3), (3.1), п. (i) предложения 1 и определения $L$.

(ii) Это следует из пп. (iii), (iv) предложения 1 , замкнутости морфизма $\pi_{8}$ и замкнутости множества $\pi_{2}^{-1}\left(G \cdot\left[x^{2}\right]\right)$.

(iii) Из формулы (4.1), очевидно, следует, что

$$
D=G \cdot S, \quad \text { где } S=\left\langle x^{8}, x^{7} y, x^{6} y^{2}, x^{5} y^{3}, x^{4} y^{4}, x^{3} y^{5}\right\rangle .
$$

Доказываемое равенство вытекает из (8.2), (9.1) и п. (i) предложения 1.

(iv) Поскольку множество $\pi_{2}^{-1}(G \cdot[x y])$ открыто в $Z$, утверждение следует из пп. (i)-(iii) и формулы (8.1).

(v) Ввиду определения множества $L$ и эквивариантности морфизма $\pi_{8}$ достаточно показать, что слой $\pi_{8}^{-1}(g)$ содержит только одну точку для форм $g$ обшего положения в $N$ (т.е. форм из некоторого непустого открытого подмножества в $N)$.

Ввиду (3.1) можно считать $g_{0}, g_{1}, g_{2}, g_{6}, g_{7}, g_{8}$ координатами на $N$. Обозначим через $(*)$ систему уравнений, полученную из (7.1) подстановкой $g_{3}=0$, $g_{4}=1, g_{5}=0$. Будем рассматривать $(*)$ как систему уравнений от $f_{0}, f_{1}, f_{2}$, считая $g_{0}, g_{1}, g_{2}, g_{6}, g_{7}, g_{8}$ параметрами. При фиксированных значениях параметров $g_{0}, g_{1}, g_{2}, g_{6}, g_{7}, g_{8}$ множество проективных решений $\left(\alpha_{0}: \alpha_{1}: \alpha_{2}\right)$ системы $(*)$ (т.е. ненулевых решений, рассматриваемых с точностью до пропорциональности) находится во взаимно однозначном соответствии с множеством точек слоя $\pi_{8}^{-1}(g)$, где $g=\left(g_{0}, g_{1}, g_{2}, g_{6}, g_{7}, g_{8}\right) \in N$. А именно, указанные $\left(\alpha_{0}: \alpha_{1}: \alpha_{2}\right) \in \mathbb{P}\left(V_{2}\right)$ 一 это в точности точки множества $\pi_{2}\left(\pi_{8}^{-1}(g)\right)$. Поэтому дело сводится к тому, чтобы показать, что для форм $g$ общего положения в $N$ число проективных решений системы $(*)$ равно 1.

Заметим, прежде всего, что $(0: 1: 0)$ является проективным решением системы $(*)$ для любой формы $g \in N$ (см. (8.3)). Рассмотрим теперь другие возможные решения. Требуется показать, что для форм $g$ общего положения в $N$ их число равно 0.

Сначала рассмотрим те из них, у которых $f_{2}=0$ и $f_{0}=1$. Подставляя $f_{2}=0$ и $f_{0}=1 \mathrm{в}(*)$, получаем систему $2 f_{1}^{2}+g_{6}=0,6 f_{1} g_{6}-7 g_{7}=0,6 f_{1}^{2} g_{6}-21 f_{1} g_{7}+$ $56 g_{8}=0$ относительно $f_{1}$, которая, очевидно, несовместна для форм $g$ общего положения в $N$.

Остается рассмотреть те решения системы $(*)$, у которых $f_{2}=1$. Подставляя $f_{2}=1$ в $(*)$, получаем следуюшую систему уравнений относительно $f_{0}$ и $f_{1}$ :

$$
\left.\begin{array}{l}
h_{1}:=56 g_{0}-21 f_{1} g_{1}+6\left(f_{0}+f_{1}^{2}\right) g_{2}+4 f_{0} f_{1}^{2}-4 f_{0}^{2}+2 f_{0}^{3} g_{6}=0, \\
h_{2}:=7 g_{1}-6 f_{1} g_{2}-8 f_{0} f_{1}-6 f_{0}^{2} f_{1} g_{6}+7 f_{0}^{3} g_{7}=0, \\
h_{3}:=2 g_{2}+4 f_{1}^{2}-4 f_{0}+6\left(f_{0}^{2}+f_{0} f_{1}^{2}\right) g_{6}-21 f_{0}^{2} f_{1} g_{7}+56 f_{0}^{3} g_{8}=0 .
\end{array}\right\}
$$

Будем обозначать через $\operatorname{Res}(p, q ; z)$ результант многочленов $p$ и $q$ от переменной $z$. Тогда

$$
R:=\operatorname{Res}\left(\operatorname{Res}\left(h_{1}, h_{2} ; f_{1}\right), \operatorname{Res}\left(h_{2}, h_{3} ; f_{1}\right) ; f_{0}\right)
$$

является таким многочленом от $g_{0}, g_{1}, g_{2}, g_{6}, g_{7}, g_{8}$, что система (9.2) совместна только при тех значениях $g_{0}, g_{1}, g_{2}, g_{6}, g_{7}, g_{8}$, которые обращают $R$ в 
нуль. Поэтому, чтобы доказать несовместность системы (9.2) для форм $g$ общего положения в $N$, нужно проверить, что $R$ - ненулевой многочлен. В свою очередь, для этого достаточно указать какой-либо конкретный набор значений параметров $g_{0}, g_{1}, g_{2}, g_{6}, g_{7}, g_{8}$, на котором $R$ не обращается в нуль. Это действительно можно сделать: например, значение $R$ при $g_{0}=g_{1}=g_{6}=g_{8}=0$, $g_{2}=g_{7}=1$ равно -2619630974070443335953257664086016 (мы проверили это двумя способами, пользуясь компьютерными программами MAGMA и MAPLE. Вычисления занимают сотые доли секунды. Отметим, что попытки проверить, что $R$ отличен от нуля, вычислив его явный вид, не заканчиваются успехом: объем вычислений оказывается слишком велик даже для мощного компьютера). Этим завершается доказательство утверждения (v).

(vi) Первое равенство вытекает из пп. (iv), (v) настоящего предложения, п. (iv) предложения 1 и теоремы о слоях морфизма. Для доказательства второго достаточно ввиду п. (iii) настоящего предложения, п. (iii) предложения 1 и теоремы о слоях морфизма указать такую форму $g \in D$, что слой $\pi_{8}^{-1}(g)$ конечен. Мы утверждаем, что $g=x^{4} y^{4}$ обладает этим свойством.

В самом деле, $g$ имеет корень кратности 4, и потому ввиду (4.1), действительно, $g \in D$. Далее, форма $g$ имеет координаты $g_{i}=0$ при $i \neq 4$ и $g_{4}=1$. Подставляя их в систему (7.1), мы получаем следующую систему уравнений на (проективные) координаты точек множества $\pi_{2}\left(\pi_{8}^{-1}(g)\right)$ :

$$
f_{0} f_{1}^{2}-f_{0}^{2} f_{2}=f_{0} f_{1} f_{2}=f_{0} f_{2}^{2}-f_{1}^{2} f_{2}=0
$$

Легко видеть, что она имеет ровно три решения: $(1: 0: 0),(0: 1: 0)$ и $(0: 0: 1)$. Значит, действительно, слой $\pi_{8}^{-1}(g)$ конечен.

ЗАмЕЧАнИЕ 3. Как видно из доказательства утверждения (vi), для некоторых форм $g \in \bar{L}$ слой $\pi_{8}^{-1}(g)$ может содержать более одной точки. В действительности существуют и такие $g \in \bar{L}$, что слой $\pi_{8}^{-1}(g)$ бесконечен: исследуя более тщательно систему (9.2) и используя компьютерные вычисления, можно показать, что форма $g$ обладает этим свойством в точности тогда, когда она содержится в множестве $E:=G \cdot C \cup G \cdot C_{+} \cup G \cdot C_{-}$, где $C:=\left\{\left(t x^{2}-y^{2}\right)^{4} / 6 t^{2} \mid\right.$ $\left.t \in \mathbb{C}^{*}\right\}$ и $C_{ \pm}:=\left\{-\left(x^{2} \pm 3 t x y-3 t^{2} y^{2}\right)^{4} / 189 t^{4} \mid t \in \mathbb{C}^{*}\right\}$. Ввиду (4.1) множество $E$ лежит в $D$. Можно показать также, что любой слой $\pi_{8}^{-1}(g), g \in E$, изоморфен $\mathbb{C}^{1}$. Эти факты не будут использованы ниже, поэтому мы опускаем их доказательство (см., однако, замечание 5).

10. Доказательство теоремы 1. (i) Это вытекает из п. (iv) предложения 2.

(ii) Это вытекает из пп. (ii), (iii), (vii) предложения 2.

(iii) Это вытекает из п. (i) доказываемой теоремы и определения (4.1).

(iv) В самом деле, $x^{4} y^{4} \in L \cap D$.

11. Доказательство теоремы 2. Из теоремы 1 следует, что $L \backslash D=\bar{L} \backslash D$. Поэтому достаточно показать, что $L^{0} \cap D=\varnothing$. Поскольку $L^{0}$ открыто в $\bar{L}$, а $D$ неприводимо и замкнуто, последнее равенство будет установлено, если мы покажем, что $\overline{D \cap L} \neq D$. 
Рассуждая от противного, предположим, что $\overline{D \cap L}=D$. По теореме Шевалле множество $L$ конструктивно. Поэтому из последнего равенства и замкнутости $D$ следует, что $D \cap L$ содержит непустое открытое подмножество множества $D$. Ввиду (9.1) его пересечение с $S$ является непустым открытым подмножеством в $S$. Следовательно,

$$
\overline{S \cap L}=S \text {. }
$$

Ввиду (9.1) можно считать $g_{0}, \ldots, g_{5}$ координатами на $S$. Обозначим через (**) систему уравнений, полученную из (7.1) подстановкой $g_{6}=g_{7}=g_{8}=0$. Рассмотрим в $\mathbb{P}\left(V_{2}\right) \times S \subset \mathbb{P}\left(V_{2}\right) \times V_{8}$ подсхему, определенную системой $(* *)$. Пусть $\Omega$ - открытое подмножество этой подсхемы, определенное условием $4 f_{0} f_{2}-f_{1}^{2} \neq 0$. Тогда из п. (i) предложения 2 следует, что

$$
S \cap L=\pi_{8}(\Omega) .
$$

Пусть $\Omega_{2}$ и $G \cdot[x y]_{2}$ - замкнутые подмножества в $\Omega$ и $G \cdot[x y]$ соответственно, заданные уравнением $f_{2}=0$.

Система (**) является линейной системой относительно $g_{0}, \ldots, g_{5}$ с коэффициентами - однородными многочленами третьей степени от $f_{0}, f_{1}, f_{2}$. Более того, она ступенчатая и обладает следующими свойствами: если $f_{2} \neq 0$, то $g_{0}$, $g_{1}, g_{2}$ - главные, а $g_{3}, g_{4}, g_{5}$ - свободные неизвестные и все коэффициенты при главных пропорциональны $f_{2}^{3}$; если же $f_{2}=0$, то $g_{0}, g_{1}, g_{2}$ - свободные, a $g_{3}, g_{4}, g_{5}$ - главные и все коэффициенты при главных пропорциональны $f_{1}^{3}$. Поскольку главные неизвестные выражаются через свободные, это означает, что при $f_{2} \neq 0$ неизвестные $g_{0}, g_{1}, g_{2}$ представляются в виде многочленов от $g_{3}, g_{4}, g_{5}, f_{0} / f_{2}, f_{1} / f_{2}$, а при $f_{2}=0$ неизвестные $g_{3}, g_{4}, g_{5}$ представляются в виде многочленов от $g_{0}, g_{1}, g_{2}, f_{0} / f_{1}$. Следовательно, $\pi_{8}\left(\Omega \backslash \Omega_{2}\right)$ и $\pi_{8}\left(\Omega_{2}\right)$ являются образами соответственно $\left(G \cdot[x y] \backslash G \cdot[x y]_{2}\right) \times \mathbb{C}^{3}$ и $\left(G \cdot[x y]_{2}\right) \times \mathbb{C}^{3}$ при некоторых морфизмах в $S$. Поскольку $\operatorname{dim}\left((G \cdot[x y]) \times \mathbb{C}^{3}\right)=5<6=\operatorname{dim} S$ (см. (9.1)), это противоречит ввиду (11.2) равенству (11.1).

ЗАмечАниЕ 4. Указанные в доказательстве морфизмы легко выписать явно. Это дает параметрическое представление множества $S \cap L$, позволяющее исследовать структуру этого множества весьма детально.

12. Для доказательства теоремы 3 нам понадобятся несколько общих фактов. В их формулировках используются следующие обозначения:

$\mathrm{I}(X)$ - мультипликативная группа регулярных обратимых функций на неприводимом алгебраическом многообразии $X$, профакторизованная по подгруппе констант;

$\mathfrak{X}(P)$ - группа характеров алгебраической группы $P$.

Если $P$ - алгебраическая группа, $Q$ - ее замкнутая подгруппа и $Z$ - алгебраическое многообразие, на котором действует $Q$, то обозначим через

$$
\pi_{P, Q, Z}: P *_{Q} Z \rightarrow P / Q
$$

однородное расслоение над $P / Q$ со слоем $Z$.

Лемма 1 [8, Theorem 1$]. \mathrm{I}(X)$ является свободной абелевой группой конечного ранга.

Лемма 2 [3, Proposition 2.3]. Пусть $X$ и $Y$ - неприводимые алгебраические многообразия $и \varphi: X \rightarrow Y$ - алгебраическое расслоение со слоем $F$, локально тривиальное в топологии Зарисского. Предположим, что база $Y$ является 
гладким, а слой $F$ - гладким рациональным многообразиями. Тогда существует следуюшая точная последовательность гомоморфизмов групп:

$$
0 \rightarrow \mathrm{I}(Y) \rightarrow \mathrm{I}(X) \rightarrow \mathrm{I}(F) \rightarrow \operatorname{Pic}(Y) \rightarrow \operatorname{Pic}(X) \rightarrow \operatorname{Pic}(F) \rightarrow 0 .
$$

Лемма $3[7$, теорема 4]. Пусть $P-$ свлзнал односвязная линейнал алгебраическая группа и $Q-$ ее алгебраическая подгруппа. Тогда существует следуюшая точная последовательность гомоморфизмов групп:

$$
\mathfrak{X}(P) \rightarrow \mathfrak{X}(Q) \rightarrow \operatorname{Pic}(P / Q) \rightarrow 0 .
$$

Лемма 4. Пусть $P$ - алгебраическая группа, $Q-$-е замкнутая подгруп$n a, V$ - конечномерный алгебраический $Q$-модуль и $V^{0}-$ непустое открытое $Q$-инвариантное подмногообразие в $V$. Предположим, что многообразие $P *_{Q} V^{0}$ изоморфно открытому подмногообразию $U^{0}$ некоторого неприводимого нормального алгебраического многообразия $U . E c л и \mathrm{I}(U)=0, \operatorname{Pic}(U)=0$ u подаруппа кручения в аруппе $\operatorname{Pic}(P / Q)$ нетривиальна, то $\operatorname{codim}_{U}\left(U \backslash U^{0}\right)=1$.

ДоказАтельство. Так как $U^{0}$ открыто в $U$, то из равенства $\operatorname{Pic}(U)=0$ следует, что $\operatorname{Pic}\left(U^{0}\right)=0$ и, значит,

$$
\operatorname{Pic}\left(P *_{Q} V^{0}\right)=0 .
$$

Рассуждая от противного, предположим, что $\operatorname{codim}_{U}\left(U \backslash U^{0}\right) \geqslant 2$. Тогда ввиду нормальности многообразия $U$ из $\mathrm{I}(U)=0$ следует, что $\mathrm{I}\left(U^{0}\right)=0$ и, значит,

$$
\mathrm{I}\left(P *_{Q} V^{0}\right)=0 .
$$

По определению $P *_{Q} V^{0}$ является геометрическим фактором по действию группы $Q$ на $P \times V^{0}$, заданному формулой $q \cdot(p, v)=\left(p q^{-1}, q \cdot v\right)$, а морфизм $\pi_{P, Q, V}$ индуцирован проекцией $P \times V^{0}$ на первый сомножитель. Хотя в общем случае эта конструкция приводит к расслоениям, локально тривиальным в этальной топологии, в нашем случае расслоение локально тривиально в топологии Зарисского. В самом деле, $P *_{Q} V^{0}$ является $P$-инвариантным открытым подмножеством в $P *_{Q} V$. Поскольку $\pi_{P, Q, V}: P *_{Q} V \rightarrow P / Q-$ (однородное) векторное расслоение над $P / Q$, утверждение следует из хорошо известного факта, что векторные расслоения, локально тривиальные в этальной топологии, автоматически локально тривиальны в топологии Зарисского.

Поскольку однородное пространство $G / H$ гладко, а многообразие $V^{0}$ гладко и рационально, мы видим, что при $X=P *_{Q} V^{0}, Y=P / Q$ и $F=V^{0}$ выполнены условия леммы 2. Поэтому, подставляя (12.2) и (12.3) в (12.1), мы получаем, что группы $\mathrm{I}\left(V^{0}\right)$ и $\operatorname{Pic}(P / Q)$ изоморфны. Но ввиду леммы 1 это противоречит условию, что $\operatorname{Pic}(P / Q)$ имеет нетривиальное кручение.

13. Доказательство теоремы 3. Предположим противное: пусть в $L^{0}$ coдержится $G$-инвариантное нормальное открытое подмножество $U$, для которого $\operatorname{Pic}(U)=0$ и $\mathrm{I}(U)=0$.

Из теоремы 2, формулы (8.1) и пп. (iii), (iv) предложения 2 следует, что

$$
\pi_{8}^{-1}(U) \subseteq \pi_{2}^{-1}(G \cdot[x y]) .
$$


Рассмотрим в $\bar{L}$ подмножество $E:=\left\{g \in \bar{L} \mid \operatorname{dim} \pi_{8}^{-1}(g)>0\right\}$. Оно замкнуто и $G$-инвариантно. Ввиду п. (iv) предложения 1 , пп. (ii), (iv) и (v) предложения 2 и теоремы о слоях морфизма имеет место неравенство

$$
\operatorname{codim}_{\bar{L}} E \geqslant 2 \text {. }
$$

Из определения множества $E$ следует, что слои морфизма

$$
\pi_{8}: \pi_{8}^{-1}(U \backslash E) \rightarrow U \backslash E
$$

конечны. Поскольку $\operatorname{char} \mathbb{C}=0$, из п. (v) предложения 2 следует, что этот морфизм является бирациональным изоморфизмом. В силу нормальности подмножества $U$ отсюда и из основной теоремы Зарисского вытекает, что (13.3) - изоморфизм.

Рассмотрим в $N$ открытое подмножество $N^{0}=N \cap(U \backslash E)$. Поскольку $L=G \cdot N$, мы имеем равенство

$$
U \backslash E=G \cdot N^{0} .
$$

Так как (13.3) является изоморфизмом, из (13.1), (8.3) и (3.1) следует, что $\pi_{8}^{-1}\left(N^{0}\right)=[x y] \times N^{0}$. Ввиду эквивариантности морфизма $\pi_{8}$ отсюда и из $(13.4)$ мы получаем, что

$$
\pi_{8}^{-1}(U \backslash E)=G \cdot\left([x y] \times N^{0}\right) .
$$

Пользуясь факториальностью кольца $\mathbb{C}[x, y]$, легко проверить, что $G$-стабилизаторы точек $[x y] \in \mathbb{P}\left(V_{2}\right)$ и $x^{4} y^{4} \in V_{8}$ совпадают с группой

$$
H=\left\{\left[\begin{array}{cc}
t & 0 \\
0 & t^{-1}
\end{array}\right],\left[\begin{array}{cc}
0 & t \\
-t^{-1} & 0
\end{array}\right] \mid t \in \mathbb{C}^{*}\right\} .
$$

Пусть $M=\left\langle x^{8}, x^{7} y, x^{6} y^{2}, x^{2} y^{6}, x y^{7}, y^{8}\right\rangle$, так что $N=x^{4} y^{4}+M$. Проекция $\pi: N \rightarrow M, x^{4} y^{4}+m \mapsto m$, является изоморфизмом. Пусть $M^{0}=\pi\left(N^{0}\right)$, так что $N^{0}=x^{4} y^{4}+M^{0}$. Многообразия $N, M, N^{0}$ и $M^{0}$ инвариантны относительно $H$, а $\pi$ является $H$-эквивариантным морфизмом.

Рассмотрим однородное расслоение $\pi_{G, H, M^{0}}: G *_{H} M^{0} \rightarrow G / H$. Легко проверить, что морфизм

$$
\alpha: G \times M^{0} \rightarrow G \cdot\left([x y] \times N^{0}\right), \quad(g, r) \mapsto\left(g \cdot[x y], g \cdot\left(x^{4} y^{4}+r\right)\right),
$$

постоянен на слоях морфизма факторизации $\pi: G \times M^{0} \rightarrow G *_{H} M^{0}$ и потому пропускается через него, т.е. $\alpha=\beta$ о $\pi$ для некоторого $G$-эквивариантного морфизма $\beta: G *_{H} M^{0} \rightarrow G \cdot\left([x y] \times N^{0}\right)$.

Морфизм $\beta$ биективен. В самом деле, поскольку $\alpha=\beta$ о $\pi$, его сюръективность следует из сюръективности морфизмов $\alpha$ и $\pi$. Инъективность же легко выводится из определений $\pi$ и $G *_{H} M^{0}$ и того, что $H$ является стабилизатором точки $[x y]$.

Равенство (13.5), изоморфизм (13.3) и нормальность подмножества $U$ показывают, что $G \cdot\left([x y] \times N^{0}\right)$ - нормальное многообразие. В силу биективности морфизма $\beta$ и основной теоремы Зарисского отсюда следует, что

$$
G *_{H} M^{0} \text { изоморфно } U \backslash E \text {. }
$$

В силу односвязности группы $G$, равенства $\mathfrak{X}(G)=0$ и леммы 3 группа $\operatorname{Pic}(G / H)$ изоморфна группе $\mathfrak{X}(H)$. Последняя же, как нетрудно вывести 
из (13.6), имеет порядок 2. С другой стороны, из (13.7), (13.2) и леммы 4 вытекает, что $\operatorname{Pic}(G / H)$ является группой без кручения. Полученное противоречие завершает доказательство теоремы 3.

ЗАмечаниЕ 5. Согласно замечанию 3 , в действительности $U \backslash E=U$. С помощью тех же рассуждений, что и в доказательстве теоремы 3 , отсюда выводится, что $L^{0}$ либо не является нормальным, либо изомофно $G *_{H} M^{0}$, где $x^{4} y^{4}+M^{0}=N \cap L^{0}$ (и, значит, гладко).

14. В первоначальном варианте работы доказывалось только, что $L$ не является аффинным открытым подмножеством в своем замыкании. Доказательство основывалось на соображениях, отличных от приведенных здесь. Мы благодарим Э. Б. Винберга, побудившего нас исследовать $L$ более подробно. Он обнаружил также ошибочное рассуждение в одной из промежуточных версий настоящей работы.

Мы также признательны Г. Кемперу за помощь при проведении вычислений и Х. Крафту за несколько замечаний.

\section{ЛитеРАТУРА}

1. Elliot E. B. An introduction to the algebra of quantics, 2nd ed. Oxford Univ Press, Oxford, 1913.

2. Fankhauser $M$. Fixed points for reductive group actions on acyclic varieties. Ann. Inst. Fourier, 45, No. 5, 1249-1281 (1995).

3. Fossum R., Iversen B. On Picard groups of algebraic fibre spaces. J. Pure Appl. Algebra, 3, 269-280 (1973).

4. Gelfand I. M., Kapranov M., Zelevinsky A. Discriminants, resultants and multidimensional determinants. Birkhäuser, Boston-Basel-Berlin, 1994.

5. Kraft $H$. Challenging problems on affine $n$-space. Séminaire Bourbaki, 47ème année, 802, 1-19 (1994-95).

6. Oliver R. Weight systems for $\mathrm{SO}(3)$-actions. Ann. of Math., 103, 637-644 (1979).

7. Попов В. Л. Группы Пикара однородных пространств линейных алгебраических групп и одномерные однородные векторные расслоения. Известия АН СССР, сер. мат., 38, 294-322 (1974).

8. Rosenlicht M. Toroidal algebraic groups. Proc. Amer. Math. Soc., 12, 984-988 (1961).

Московский независимый университет, email: katsylo@katsylo.mccme.ru

Московский государственный институт электроники и математики, email: popov@ppc.msk.ru
Поступило в редакцию 16 сентября 1998 г. 